Meta

Journal des traducteurs

Translators' Journal

\title{
Un estudio experimental sobre la adquisición de la competencia cultural en la formación de traductores. Resultados de un estudio piloto
}

\section{Christian Olalla-Soler}

Volume 62, numéro 2, août 2017

URI : https://id.erudit.org/iderudit/1041032ar

DOI : https://doi.org/10.7202/1041032ar

Aller au sommaire du numéro

Éditeur(s)

Les Presses de l’Université de Montréal

ISSN

0026-0452 (imprimé)

1492-1421 (numérique)

Découvrir la revue

Citer cet article

Olalla-Soler, C. (2017). Un estudio experimental sobre la adquisición de la competencia cultural en la formación de traductores. Resultados de un estudio piloto. Meta, 62(2), 435-460. https://doi.org/10.7202/1041032ar

\section{Résumé de l'article}

Cet article présente les principaux résultats de la phase pilote d'une étude expérimentale sur l'acquisition de la compétence culturelle dans la formation des traducteurs. Cette étude s'inscrit dans le cadre des recherches du Groupe PACTE (Processus d'Acquisition de la Compétence de Traduction et Évaluation) sur l'acquisition de la compétence de traduction. L’approche méthodologique retenue a été testée à partir d'un échantillon de cinq étudiants en dernière année de licence de traduction et d'interprétation à l'Université Autonome de Barcelone. Les étudiants avaient pour langue maternelle l'espagnol ou le catalan et pour deuxième langue étrangère, l'allemand. Des données ont été recueillies pour vérifier la pertinence des critères de sélection de l'échantillon, l'adéquation des problèmes de traduction sélectionnés pour une analyse plus approfondie, le fonctionnement des outils de collecte de données, ainsi que la durée et l'ordre des tâches expérimentales. Les résultats montrent que les outils ont permis de recueillir des données pertinentes, que l'échantillon a été constitué à partir d'une population normale et que les problèmes de traduction sélectionnés pour une analyse plus approfondie étaient adaptés. Il convient toutefois de réviser les tâches expérimentales et de collecter des données supplémentaires sur les aspects culturels.
Ce document est protégé par la loi sur le droit d'auteur. L’utilisation des services d’Érudit (y compris la reproduction) est assujettie à sa politique d'utilisation que vous pouvez consulter en ligne.

https://apropos.erudit.org/fr/usagers/politique-dutilisation/ 


\title{
Un estudio experimental sobre la adquisición de la competencia cultural en la formación de traductores. Resultados de un estudio piloto
}

\author{
CHRISTIAN OLALLA-SOLER \\ Universitat Autònoma de Barcelona, Bellaterra, España \\ christian.olalla@uab.cat
}

\section{RÉSUMÉ}

Cet article présente les principaux résultats de la phase pilote d'une étude expérimentale sur l'acquisition de la compétence culturelle dans la formation des traducteurs. Cette étude s'inscrit dans le cadre des recherches du Groupe PACTE (Processus d'Acquisition de la Compétence de Traduction et Évaluation) sur l'acquisition de la compétence de traduction. L'approche méthodologique retenue a été testée à partir d'un échantillon de cinq étudiants en dernière année de licence de traduction et d'interprétation à l'Université Autonome de Barcelone. Les étudiants avaient pour langue maternelle l'espagnol ou le catalan et pour deuxième langue étrangère, l'allemand. Des données ont été recueillies pour vérifier la pertinence des critères de sélection de l'échantillon, l'adéquation des problèmes de traduction sélectionnés pour une analyse plus approfondie, le fonctionnement des outils de collecte de données, ainsi que la durée et l'ordre des tâches expérimentales. Les résultats montrent que les outils ont permis de recueillir des données pertinentes, que l'échantillon a été constitué à partir d'une population normale et que les problèmes de traduction sélectionnés pour une analyse plus approfondie étaient adaptés. Il convient toutefois de réviser les tâches expérimentales et de collecter des données supplémentaires sur les aspects culturels.

\begin{abstract}
This article presents the main results obtained in the pilot test of an experimental study on the acquisition of cultural competence in translator training. This study is part of PACTE's (Process in the Acquisition of Translation Competence and Evaluation) research into the acquisition of translation competence. The research design of this study was pilot tested with a sample of five trainee translators in the final year of the degree in Translation and Interpreting at the Universitat Autònoma de Barcelona. Subjects' L2 was German, and Spanish or Catalan was their native language. Data was gathered to test the suitability of the sample-selection criteria, the translation problems that were selected for deeper analysis, the performance of the data collection instruments and the length and order of the experimental tasks. Results show that the data collection instruments gathered relevant data, the selected sample was drawn from a normal population and that the translation problems selected for deeper analysis were suitable. However, the experimental tasks have to be revised and more data on cultural aspects has to be collected.
\end{abstract}

\section{RESUMEN}

En este artículo se presentan los principales resultados del estudio piloto de un estudio experimental sobre la adquisició de la competencia cultural en la formación de traductores. Este estudio se enmarca en la investigación sobre adquisición de la competencia traductora del Grupo PACTE (Proceso de Adquisición de la Competencia Traductora y Evaluación). El diseño experimental del estudio se pilotó con una muestra de cinco estudiantes con alemán como segunda lengua extranjera del último curso del Grado en Traducción e Interpretación de la Universitat Autònoma de Barcelona. La lengua materna 
de los estudiantes era el español o el catalán. Se recogieron datos para comprobar la adecuación de los criterios de selección de la muestra, la idoneidad de los problemas de traducción seleccionados para el análisis, el funcionamiento de los instrumentos de recogida de datos y la duración y el orden de las tareas experimentales. Los resultados han demostrado que los instrumentos recogen datos pertinentes, la muestra seleccionada era representativa de la población de estudio y los problemas de traducción eran útiles para el análisis. Sin embargo, las tareas experimentales deben ser revisadas y deben recogerse más datos sobre aspectos culturales.

\section{MOTS-CLÉS/KEYWORDS/PALABRAS CLAVE}

acquisition de la compétence culturelle, étude pilote, étude expérimentale, compétence de traduction, formation des traducteurs

cultural competence acquisition, pilot test, experimental study, translation competence, translator training

adquisición de la competencia cultural, estudio piloto, estudio experimental, competencia traductora, formación de traductores

\section{Introducción}

El objetivo de este artículo es presentar los resultados obtenidos en el estudio piloto de un experimento sobre la adquisición de la competencia cultural en estudiantes de traducción con el fin de evaluar la metodología presentada en Olalla-Soler (2015). El estudio se pilotó con una muestra de cinco estudiantes de último curso del Grado en Traducción e Interpretación de la Universitat Autònoma de Barcelona (UAB) con alemán como segunda lengua extranjera.

Se recogieron datos para probar los siguientes aspectos: a) la pertinencia de los criterios de selección de la muestra, b) la adecuación de los problemas de traducción seleccionados para el análisis, c) el funcionamiento de los instrumentos de recogida de datos y d) la duración y el orden de las tareas experimentales.

El pilotado también sirvió para seleccionar las variables y los indicadores de los experimentos de competencia traductora (CT) y de adquisición de la competencia traductora (ACT) de PACTE (2014) que se utilizarán en la versión revisada de este experimento. A continuación enumeramos las variables y los indicadores que se tratan en el presente artículo. En la enumeración se detalla también la sección en la cual se presenta cada variable.

1) Variable «Conocimientos culturales» (sección 4.2):

a. Índice de conocimientos declarativos sobre la cultura alemana;

b. Cantidad de puntos ricos identificados como culturemas;

c. Cantidad de puntos ricos caracterizados de acuerdo con la caracterización de los expertos;

d. Índice de conocimientos culturales relacionados con el tema del texto.

2) Variable «Conocimientos de traducción» (sección 4.3):

a. Índice de dinamismo de los conocimientos de traducción;

b. Coeficiente de coherencia de los conocimientos de traducción;

3) Variable «Proyecto traductor» (sección 4.4):

a. Índice de dinamismo del proyecto traductor global;

b. Índice de dinamismo del proyecto traductor de los problemas de traducción;

c. Coeficiente de coherencia del proyecto traductor. 
4) Variable «Identificación y resolución de problemas de traducción» (sección 4.5):

a. Identificación y resolución de dificultades causadas por culturemas;

b. Índice de percepción de la dificultad de la traducción;

c. Índice de identificación de los problemas de traducción de índole cultural;

d. Caracterización de problemas de traducción de índole cultural;

e. Coeficiente de satisfacción con la traducción.

5) Variable «Toma de decisiones» (sección 4.6):

a. Secuencias de acciones.

6) Aceptabilidad (indicador transversal) (sección 4.1).

Las variables «Eficacia del proceso traductor» $\mathrm{y}$ «Uso de recursos instrumentales», que se utilizaron en los experimentos de PACTE de CT (2009) y ACT (2014), no se incluyen en el estudio. La variable «Eficacia del proceso traductor» se centra en la relación entre el tiempo dedicado a completar la traducción y la aceptabilidad de las soluciones. En nuestro estudio, el tiempo no es una variable específica de la competencia cultural. La variable «Uso de recursos instrumentales» se centra en las estrategias de documentación utilizadas durante la traducción. Esta información se recoge parcialmente en la variable «Toma de decisiones», ya que las consultas se categorizan de acuerdo con el grado de esfuerzo cognitivo (3.2. Variables).

\section{Conceptos teóricos de la competencia cultural del traductor}

En esta sección se presentan los conceptos teóricos principales del estudio: el concepto de cultura, el modelo de competencia traductora de PACTE y nuestro modelo de competencia cultural del traductor.

\subsection{El concepto de cultura}

En Olalla-Soler (2015) revisamos los conceptos de cultura que se han propuesto en Antropología desde la Ilustración hasta el materialismo cultural y concluimos que la cultura se ha descrito principalmente desde dos enfoques diferentes aunque complementarios: la cultura como proceso y el relativismo cultural.

Nuestra propuesta de definición de cultura recoge los dos enfoques antropológicos anteriormente nombrados, aunque matizados y de acuerdo con los principios generales de la Antropología contemporánea (Harris 2011: 28-42; Olalla-Soler 2015):

Una cultura es un sistema de normas, valores, estándares y modelos de comportamiento que se adquiere socialmente, se comparte en un grupo de individuos y se desarrolla en un contexto cognitivo. Mediante este sistema, el grupo de individuos percibe y modifica el medio natural y artificial de manera determinada, y desarrolla subsistemas para la organización del comportamiento, del medio natural, del patrimonio, de las estructuras sociales y de las necesidades lingüísticas y comunicativas. La cultura evoluciona constantemente mediante el contacto con otros sistemas culturales y a causa de los cambios que percibe en el medio natural. (Olalla-Soler 2015: 90, traducción del autor)

La cultura sirve como sistema de cohesión entre individuos, debido a que guía los procesos cognitivos de toma de decisiones y de percepción, valoración e interpretación del mundo y de otras culturas para el desarrollo de una realidad compartida a nivel colectivo aunque desarrollada de manera individual. También favorece socialmente ciertas actitudes o comportamientos. 


\subsection{La competencia traductora}

Nuestro estudio se basa en el modelo de competencia traductora de PACTE (2003), ya que el grupo propone una definición que consideramos pertinente, la operacionalización del modelo es muy detallada y, además, este modelo ha sido validado empíricamente (Hurtado Albir 2017).

PACTE define la competencia traductora como «the underlying system of knowledge required to translate», es «(a) expert knowledge; (b) predominantly procedural» (PACTE 2003: 58), y «(c) [it] comprises different inter-related subcompetences; and (d) includes a strategic component which is of particular importance» (PACTE 2009: 208). Las subcompetencias que componen la competencia traductora son la bilingüe, la extralingüística, la de conocimientos de traducción, la instrumental, la estratégica y los componentes psicofisiológicos (PACTE 2003: 58-59). En la subcompetencia extralingüística se incluye la competencia cultural del traductor.

\subsection{La competencia cultural del traductor}

En los Estudios Interculturales, la competencia intercultural (algunos autores utilizan la denominación cultural o bicultural, aunque no son sinónimos intercambiables) se ha definido generalmente como el uso de los conocimientos sobre la cultura propia y los de la cultura extranjera para alcanzar un objetivo específico (la comunicación entre culturas, la integración social o las negociaciones entre empresas, entre otros) utilizando habilidades específicas y desarrollando actitudes que faciliten el entendimiento, la concienciación cultural y la interacción entre culturas (Fantini 1995; Byram 1997; King y Baxter Magolda 2005; Deardorff 2006; Rathje 2007; Arasaratnam 2007 o Kupka 2008, entre otros).

Cada investigador ha establecido competencias, habilidades y actitudes de acuerdo con las necesidades específicas del campo de aplicación de su modelo. Spitzberg y Chagnon (2010) recopilaron en un estudio una gran cantidad de modelos de competencia cultural o intercultural y enumeraron las competencias, habilidades y actitudes que se mencionaban. Obtuvieron una lista de 325 elementos distintos. Esto es un indicador claro de que los investigadores todavía están lejos de proponer y validar un modelo de competencia cultural general aplicable a cualquier campo.

Para desarrollar un modelo de competencia cultural aplicable a la Traductología, partimos de:

- la Antropología. Utilizamos los ámbitos culturales estudiados en Antropología (Harris 2011) conceptualizados en nuestro modelo de cultura;

- la Traductología. Partimos del modelo empíricamente validado de competencia traductora de PACTE;

- los Estudios Interculturales. Empleamos las cuatro dimensiones de la inteligencia cultural, propuestas y validadas empíricamente por Ang, Dayne y Tan (2007), y las cinco dimensiones de la eficacia multicultural, propuestas y validadas por van der Zee y van Oudenhoven (2000). La inteligencia cultural se define como «the capability to function effectively in culturally diverse settings» (Ang, Van Dyne et al. 2007: 337). La eficacia multicultural se concibe como la capacidad de desenvolverse de manera satisfactoria y a la vez ser capaz de adaptarse a un contexto cultural nuevo (van der Zee and van Oudenhoven 2000: 292-293). 
Nuestra definición de competencia cultural del traductor se basa en la definición general que hemos comentado en el primer párrafo de esta sección. Así, pues, definimos la competencia cultural del traductor como:

las habilidades del traductor para movilizar y contrastar los conocimientos sobre la cultura de partida y los de la cultura de llegada en relación con un fenómeno cultural percibido en el texto de partida, con el fin de llegar a una solución aceptable en el texto meta. La competencia cultural del traductor está en constante relación con las subcompetencias que forman la competencia traductora. (Olalla-Soler 2015: 94, traducción del autor)

La competencia cultural se desglosa en las siguientes subcompetencias:

- conocimientos culturales. Los conocimientos sobre las dos culturas involucradas en el acto traductor son indispensables para identificar los referentes culturales en el texto de partida e interpretarlos de acuerdo con los sistemas de las culturas de partida y de llegada. Cuando el traductor tiene deficiencias en sus conocimientos culturales, puede activar otras subcompetencias de la competencia traductora para solucionar este problema, como la instrumental. Los conocimientos de las culturas de partida y de llegada están relacionados con: a) la organización del patrimonio: personajes históricos y de ficción, hechos históricos, religiones, festividades, creencias populares, folklore, monumentos, bellas artes, juegos, herramientas y objetos, instrumentos musicales, técnicas ganaderas y agrícolas, urbanismo, estrategias militares, medios de transporte, etc., b) la organización del comportamiento: cortesía, modales en la mesa, moda, valores morales, gestos y salutaciones, etc., c) las necesidades lingüísticas y comunicativas: transliteraciones, proverbios, frases hechas, interjecciones, blasfemias, insultos, etc., d) la organización del medio natural: flora, fauna, clima, fenómenos atmosféricos, paisajes, topónimos, etc., e) la organización de la sociedad: sistemas legales, políticos y económicos, educación, calendarios, unidades de medida, etc., y f) las normas, estándares, valores y modelos comportamentales centrales que regulan el sistema cultural;

- habilidades. Pueden ser habilidades relacionadas con la adquisición de conocimientos culturales o habilidades contrastivas. Las habilidades de adquisición de conocimientos se centran en la adquisición, almacenamiento y recuperación eficaces de los conocimientos culturales. Las habilidades contrastivas están vinculadas a las estrategias contrastivas entre las culturas de partida y de llegada en relación con una referencia cultural. También están vinculadas a la movilización de los conocimientos culturales y la aplicación de estrategias para solucionar adecuadamente problemas de traducción de índole cultural;

- componentes actitudinales. Las actitudes favorecen el entendimiento entre culturas y ayudan en la toma de conciencia de la influencia de la cultura propia en la percepción, valoración e interpretación de otras culturas. Distinguimos las siguientes actitudes: a) empatía cultural: empatía con los sentimientos, los pensamientos y los comportamientos de los miembros de otros grupos culturales, b) actitud abierta: actitud libre de prejuicios hacia los miembros de otros grupos culturales y hacia otras normas y valores, c) iniciativa social: la tendencia a ser activo en situaciones sociales, y d) flexibilidad: el uso de distintas estrategias para evitar situaciones de riesgo en entornos multiculturales.

La competencia cultural está vinculada a la competencia traductora y a las subcompetencias que la forman. Si el traductor no puede recuperar ningún conocimiento cultural en relación con una referencia cultural, puede activarse la subcompetencia instrumental para adquirir la información necesaria. 
También podrían activarse otras subcompetencias de la competencia traductora, como la bilingüe si el traductor debe solucionar algún problema relacionado con la comprensión o reexpresión de la lengua o de aspectos sociolingüísticos o textuales. Como en el proceso de resolución de cualquier problema de traducción, el resto de subcompetencias se activan de acuerdo con las necesidades específicas del traductor. La subcompetencia estratégica regula el vínculo entre la competencia traductora y la competencia cultural.

La definición de la competencia cultural se ha operacionalizado en la Figura 1.

FIGURA 1

El modelo de competencia cultural

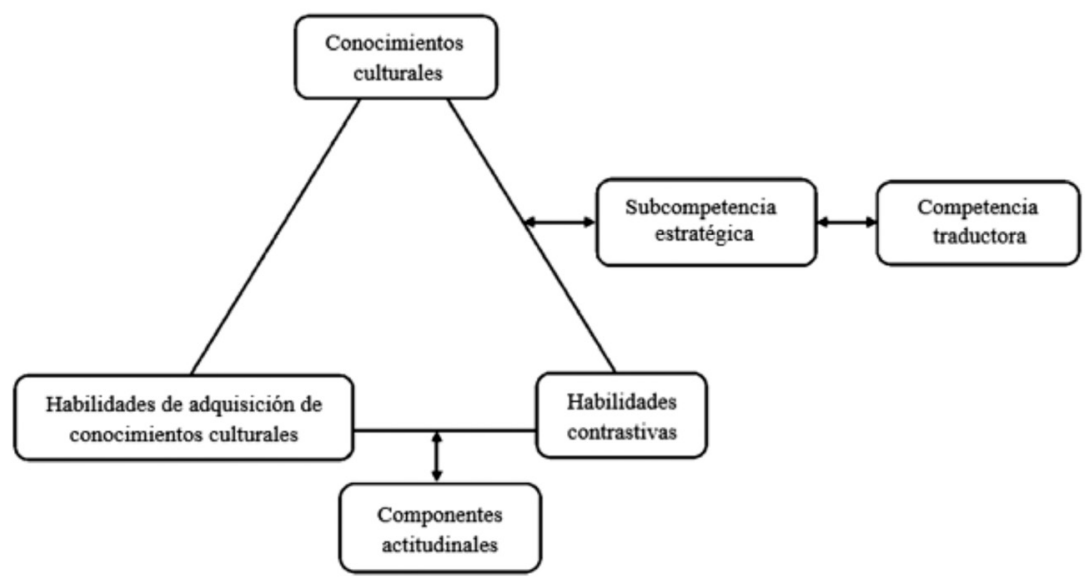

\section{Diseño metodológico del estudio}

Este estudio se basa en el diseño experimental de la investigación de PACTE en los experimentos de CT y de ACT, realizados de octubre de 2005 a octubre de 2006 y en 2011 respectivamente. El presente estudio también es un simulacro de estudio longitudinal (PACTE 2014).

La investigación de PACTE se centra en varios tipos de problemas prototípicos de traducción (puntos ricos); en nuestro caso, el diseño se ha adaptado al objetivo del estudio: los problemas de traducción de índole cultural.

Los puntos ricos, que se definen como segmentos del texto de partida que contienen problemas de traducción prototípicos (PACTE 2009), se usan también en el presente estudio para facilitar el proceso de recogida de datos. En nuestro experimento los puntos ricos son culturemas de distinta naturaleza. Para definir culturema, usamos la definición de Molina, basada en Vermeer (1983) y en Nord (1994, 1997). El concepto de culturema proviene de Poyatos (1976) y fue expandido por Oksaar en su trabajo sobre multilingüismo (1988). Molina lo define de la siguiente manera:

[...] un elemento verbal o paraverbal que posee una carga cultural específica en una cultura y que al entrar en contacto con otra cultura a través de la traducción puede provocar un problema de índole cultural entre los textos origen y meta (Molina 2001: 89). 
De esta definición destacamos que a) los culturemas existen a causa de un trasvase cultural, b) los culturemas no son universales, y c) la función de un culturema depende del contexto en el cual se percibe. Los culturemas que aparecen en el texto y los que se han seleccionado como puntos ricos se han categorizado de acuerdo con la clasificación de Molina (2001: 91-95): medio natural, patrimonio cultural, cultura social y cultura lingüística.

\subsection{Hipótesis}

Las hipótesis teóricas que se pretenden comprobar con este estudio experimental son tres:

- La competencia cultural del traductor son las habilidades del traductor para movilizar y contrastar sus conocimientos sobre la cultura de partida y los de la cultura de llegada en relación con un fenómeno cultural que se percibe en el texto de partida con el fin de llegar a una solución aceptable en el texto meta.

- La competencia cultural del traductor está vinculada a las otras subcompetencias que forman la competencia traductora.

- A medida que se adquiere la competencia cultural, los problemas de traducción de índole cultural se resuelven con mayor aceptabilidad.

Cada hipótesis teórica se divide en hipótesis empíricas. Dado que el objetivo del estudio piloto no era testar las hipótesis empíricas (y en el caso de la tercera no es posible hacerlo con los datos de un único grupo de muestra), no se han incluido en el presente artículo. Sin embargo, pueden leerse en Olalla-Soler (2015).

\subsection{Variables}

Para la prueba piloto, se seleccionaron una variable independiente y cinco variables dependientes. La variable independiente fue el grado de pericia en traducción, definida como años de formación en traducción. Para la seleccionar la muestra y mantener un grado de pericia homogéneo, se fijaron los siguientes criterios: 1) los estudiantes debían tener 21 o 22 años; 2) no debían tener el alemán como lengua materna; 3) debían tener el español o el catalán como lengua materna; 4) no debían haber estudiado alemán antes de empezar la carrera, y 5) no debían haber realizado una estancia de más de tres meses en algún país de habla alemana. Todos los sujetos pertenecían al cuarto curso del grado.

Las variables dependientes que se utilizan en el pilotado del experimento son las siguientes:

- «Conocimientos culturales». Los conocimientos declarativos y operativos sobre las culturas de partida y de llegada que posee el traductor. Está vinculada a la competencia cultural.

- «Conocimientos de traducción». Los conocimientos implícitos del sujeto sobre los principios de la traducción y sobre aspectos de la profesión. Está vinculada a la subcompetencia de conocimientos de traducción.

- «Proyecto traductor». La representación mental y las expectativas del traductor de cómo debería ser la traducción de un texto. Está vinculada a la subcompetencia estratégica.

- «Identificación y resolución de problemas de traducción de índole cultural». La identificación y resolución de las dificultades causadas por culturemas a la hora de 
realizar una tarea de traducción. Está vinculada a las subcompetencias estratégica y de conocimientos de traducción.

- «Toma de decisiones». Las decisiones tomadas durante el proceso traductor que afectan al uso de recursos cognitivos automatizados y no automatizados (apoyo interno) y el uso de recursos de documentación (apoyo externo) (Alves 1995 1997).

La aceptabilidad es el indicador transversal que sirve para medir la calidad de las traducciones propuestas a los culturemas. Definimos aceptabilidad ${ }^{1}$ de la siguiente manera: "whether or not the solution effectively communicates (a) the meaning of the source text; (b) the function of the translation (within the context of the translation brief, the readers' expectations, genre conventions in the target culture); and (c) makes use of appropriate language» (PACTE 2011b).

En la sección de resultados, se presenta una tabla para cada variable donde se presenta la definición de la variable, los indicadores medidos y los instrumentos de recogida de datos utilizados.

\subsection{Recogida de datos}

Para obtener los datos necesarios para el análisis, se han utilizado seis tipos de instrumentos de recogida de datos. El primer tipo es el texto original que los estudiantes debían traducir. Se trata de un artículo de periódico que apareció en Die Zeit y trataba de la comercialización de los símbolos de la antigua República Democrática Alemana. El texto fue sometido a dos juicios de expertos y se seleccionaron cinco culturemas como puntos ricos:

- Ostalgie (cultura lingüística): parte del título del artículo y referencia al tema basado en un neologismo marcado culturalmente;

- Spreemetropole (medio natural): referencia a Berlín a través de la mención al río que cruza la ciudad;

- Grenzeruniformen (patrimonio cultural): juego de contrastes entre la referencia a los soldados que vigilaban el Muro de Berlín (percibido como muy negativo por los berlineses) y la parodia que representan unos actores disfrazados de soldados del muro para los turistas;

- Arbeiter- und Bauernstaat (cultura social): referencia al comunismo utilizado como sinónimo para la antigua República Democrática de Alemania;

- Unrechtstaat [...] DDR-Regim [...] SED-Diktatur (punto especialmente rico): caracterización del gobierno de la antigua República Democrática de Alemania y del Partido Socialista Unificado de Alemania con sinónimos con connotaciones negativas.

El segundo tipo lo componen las traducciones del texto seleccionado que realizan los sujetos. El tercero es la grabación de las acciones que los sujetos hacen en el ordenador mediante un software de grabación de pantalla.

Cuatro cuestionarios componen el cuarto tipo de instrumentos. Cada cuestionario tiene un objetivo específico: en el cuestionario inicial se recoge información de la muestra, en el cuestionario de conocimientos declarativos sobre cultura alemana se recogen datos sobre los conocimientos de los sujetos en este ámbito, en el cuestionario de problemas de traducción se recogen datos sobre los problemas que los sujetos han intentado resolver en la traducción y, finalmente, en el cuestionario de conocimientos culturales se recoge información sobre la concepción de la traducción que los sujetos poseen. 
El quinto tipo de instrumentos es un documento para la identificación y caracterización de los culturemas que incluye tareas previas a la traducción. El sexto y último tipo es la observación directa, que tiene por objetivo asegurar que todos los datos se recopilan correctamente.

En Olalla-Soler (2015) puede encontrarse más información sobre el proceso de diseño y de validación de cada uno de los instrumentos mencionados.

\subsection{Muestra}

En la prueba piloto participaron cinco estudiantes de cuarto curso del grado en Traducción e Interpretación de la UAB.

La segunda lengua extranjera de los sujetos era el alemán. El 100\% era hablante nativo de español y un $40 \%$ era bilingüe con el catalán. Todos los sujetos tenían entre 21 y 22 años. Ningún sujeto ha estudiado alemán antes de la carrera. Durante sus estudios universitarios, un $80 \%$ estudió alemán fuera de las asignaturas de la carrera (un 20\% en escuelas de idiomas, un 60\% en estancias en Alemania de menos de tres meses y el $20 \%$ restante en clases particulares). Solo un $20 \%$ ha trabajado como traductor profesional.

\subsection{Tareas experimentales}

Después de rellenar el cuestionario de selección de la muestra, los sujetos realizaron las siguientes tareas experimentales: 1) identificaron y caracterizaron los culturemas del texto (15 minutos); 2) tradujeron el texto (1 hora y 30 minutos); 3) cumplimentaron el cuestionario de problemas de traducción (10 minutos); 4) cumplimentaron el cuestionario de conocimientos culturales (5 minutos) y 5) cumplimentaron el cuestionario de conocimientos de traducción (5 minutos).

La prueba piloto duró dos horas y quince minutos y se realizó en una sala con ordenadores conectados a internet en la Facultad de Traducción e Interpretación de la UAB. El experimento revisado se realizó en julio de 2015.

\section{Resultados}

En esta sección se presentan los resultados ordenados por variable e indiciador con una breve descripción del método de cálculo de cada indicador.

En los resultados se reporta la media y la desviación estándar (DE). Este estadístico descriptivo mide la dispersión de los datos de la muestra alrededor de la media.

En los indicadores que no están relacionados directamente con el texto y los puntos ricos seleccionados (índice de dinamismo de los conocimientos de traducción, coeficiente de coherencia de los conocimientos de traducción y índice de dinamismo del proyecto traductor global), se comparan los resultados obtenidos con los de la muestra de cuarto curso del experimento de ACT de PACTE.

\subsection{Aceptabilidad}

Para el cálculo de la aceptabilidad, en cada punto rico se asigna una de las siguientes categorías de aceptabilidad al sentido, a la función y al uso de la lengua: aceptable 
(A), semiaceptable (SA) y no aceptable (NA). Al combinar las categorías de sentido, función y lengua se obtienen veintisiete permutaciones posibles para un punto rico. Los resultados de las permutaciones se clasifican en soluciones aceptables, semiaceptables o no aceptables. A las soluciones aceptables se les asigna un valor numérico de 1, a las semiaceptables de 0,5 y a las no aceptables de 0 . En la Tabla 1 se presentan algunas de las permutaciones.

TABLA 1

Permutaciones de aceptabilidad

\begin{tabular}{|c|c|c|c|c|}
\hline Sentido & Función & Lengua & Categoría & Valor numérico \\
\hline A & A & A & & \\
\cline { 1 - 2 } A & A & SA & \multirow{2}{*}{ A } & 1 \\
\hline A & SA & A & & \\
\hline SA & A & A & & \\
\cline { 1 - 2 } A & SA & SA & & \multirow{2}{*}{ SA } \\
A & A & NA & & \\
\hline A & SA & NA & & \\
\hline Etc. & & & & \\
\hline
\end{tabular}

La aceptabilidad de los puntos ricos (Tabla 2) va desde el 0,00 (en Grenzeruniformen) hasta el 0,60 (en Ostalgie y Spreemetropole). Todos los culturemas presentan una dificultad de moderada a alta. Las desviaciones estándar van desde el 0,00 hasta el 0,58 . Esto indica que los sujetos no son homogéneos a la hora de resolver estos puntos ricos. En el experimento es necesario identificar las causas de esta variabilidad.

La aceptabilidad media del grupo experimental es baja $(0,37)$, aunque la variabilidad es alta (DE: 0,20). Una posible explicación es que la subcompetencia bilingüe de los sujetos no está suficientemente desarrollada como para entender el texto sin dificultades y por tanto necesitan más tiempo para resolver estas dificultades con la subcompetencia instrumental.

TABLA 2

Aceptabilidad

\begin{tabular}{|l|c|c|}
\hline & Media & DE \\
\hline Ostalgie (cultura lingüística) & 0,60 & 0,55 \\
\hline Spreemetropole (medio natural) & 0,60 & 0,55 \\
\hline Grenzeruniformen (patrimonio cultural) & 0,00 & 0,00 \\
\hline Arbeiter- und Bauernstaat (cultura social) & 0,33 & 0,58 \\
\hline Punto especialmente rico & 0,30 & 0,48 \\
\hline Aceptabilidad del grupo experimental & 0,37 & 0,20 \\
\hline
\end{tabular}

\subsection{Variable «Conocimientos culturales»}

En la Tabla 3 presentamos la definición, los indicadores medidos y los instrumentos utilizados en la variable «Conocimientos culturales». 
TABLA 3

Definición, indicadores e instrumentos de la variable «Conocimientos culturales»

\begin{tabular}{|l|l|}
\hline \multicolumn{2}{|c|}{ CONOCIMINENTOS CULTURALES } \\
\hline definición & $\begin{array}{l}\text { Los conocimientos declarativos y operativos sobre las culturas de partida y de } \\
\text { llegada que posee el traductor. }\end{array}$ \\
\hline indicadores & $\begin{array}{l}\text { - Índice de conocimientos declarativos sobre la cultura alemana: los conocimientos } \\
\text { declarativos de la cultura alemana de los sujetos; } \\
\text { - Cantidad de puntos ricos identificados como culturemas; } \\
\text { - Cantidad de puntos ricos caracterizados de acuerdo con la caracterización de los } \\
\text { expertos; } \\
\text { - Indice de conocimientos culturales relacionados con el tema del texto; } \\
\text { - Aceptabilidad. }\end{array}$ \\
\hline instrumentos & $\begin{array}{l}\text { Cuestionario de conocimientos declarativos sobre la cultura alemana, documento } \\
\text { para la identificación y caracterización de culturemas y las traducciones }\end{array}$ \\
\hline
\end{tabular}

4.2.1. Indice de conocimientos declarativos sobre la cultura alemana

El rango de este índice va del 0 (nivel bajo de conocimientos) al 1 (nivel alto de conocimientos). Los datos se obtienen del cuestionario conocimientos declarativos sobre la cultura alemana. Se asigna un 1 a las respuestas correctas y un 0 a las incorrectas. Se calcula la media de cada ámbito cultural y después se calcula la media de los cuatro ámbitos que cubre el cuestionario.

Las medias del índice de conocimientos declarativos (Tabla 4) van desde el 0,42 hasta el 0,55. No se observan diferencias relevantes entre ámbitos. En la organización del mundo natural se registra la mayor desviación estándar $(0,35)$. En el resto de ámbitos, la variabilidad es baja (desde 0,06 hasta 0,18 ).

El índice del grupo experimental es de 0,50 (nivel medio de conocimientos). El grupo es bastante homogéneo ya que la variabilidad es baja $(0,09)$. Aunque el nivel medio de conocimientos y la homogeneidad del grupo experimental son buenos indicadores de la validez de este instrumento, ésta debe evaluarse más detenidamente (Olalla-Soler y Neunzig, en proceso).

TABLA 4

Índice de conocimientos declarativos sobre la cultura alemana

\begin{tabular}{|l|c|c|}
\hline & Media & DE \\
\hline Organización del medio natural & 0,55 & 0,35 \\
\hline Organización del patrimonio cultural & 0,42 & 0,14 \\
\hline Organización de la sociedad & 0,50 & 0,06 \\
\hline Necesidades lingüísticas y comunicativas & 0,53 & 0,18 \\
\hline Índice del grupo experimental & 0,50 & 0,09 \\
\hline
\end{tabular}

\subsubsection{Cantidad de puntos ricos identificados como culturemas}

La cantidad de puntos ricos identificados como culturemas se indica en un índice que va del 0 (identificación baja) al 1 (identificación alta). Los datos se obtienen del documento para la identificación y caracterización de los culturemas. Para el cálculo, se cuenta el número de puntos ricos identificados como culturemas y el valor obtenido se codifica en una escala del 0 al 1.

Los resultados de la identificación de los puntos ricos como culturemas (Tabla 5) muestran que solo la mitad de los puntos ricos se han identificado como culturemas 
$(0,55)$. La desviación estándar es alta $(0,20)$. El grupo no es homogéneo a la hora de identificar culturemas. Para el experimento será relevante identificar las causas de esta heterogeneidad mediante el cruzamiento de este indicador con otros.

TABLA 5

Cantidad de puntos ricos identificados como culturemas

\begin{tabular}{|l|c|}
\hline & Índice \\
\hline Media & 0,55 \\
\hline $\mathrm{DE}$ & 0,21 \\
\hline
\end{tabular}

4.2.3. Cantidad de puntos ricos caracterizados de acuerdo con la caracterización de los expertos

Los resultados se presentan en un índice comprendido entre el 0 (caracterización baja) y el 1 (caracterización alta). Los datos se obtienen del documento para la identificación y caracterización de los culturemas. Para el cálculo, se cuenta el número de puntos ricos caracterizados adecuadamente según los expertos y el valor obtenido se codifica en una escala del 0 al 1.

En los resultados de este indicador (Tabla 6), se observa el mismo patrón que en el indicador cantidad de puntos ricos identificados como culturemas: se caracterizan adecuadamente alrededor de la mitad de puntos ricos $(0,45)$ y la variabilidad es alta $(0,27)$. En el experimento deberá cruzarse este indicador con otros para explicar la variabilidad.

TABLA 6

Cantidad de puntos ricos caracterizados de acuerdo con la caracterización de los expertos

\begin{tabular}{|l|c|}
\hline & Índice \\
\hline Media & 0,45 \\
\hline DE & 0,27 \\
\hline
\end{tabular}

\subsection{4. Índice de conocimientos culturales relacionados con el tema del texto}

El rango de este índice va del 0 (nivel bajo de conocimientos) al 1 (nivel alto de conocimientos). Los datos se obtienen del documento para la identificación y caracterización de los culturemas. Se asigna un valor numérico a la calidad de la explicación de los puntos ricos $(1=$ el punto rico está explicado correctamente; $0,5=$ se explica el punto rico parcialmente o bien falta información, y 0 = la explicación del punto rico es incorrecta). Con estos datos se calcula la media.

Aunque la desviación estándar en el índice de conocimientos culturales era baja $(0,09)$, es alta $(0,34)$ en el índice de conocimientos culturales relacionados con el tema del texto (Tabla 7).

TABLA 7

Índice de conocimientos culturales relacionados con el tema del texto

\begin{tabular}{|l|c|}
\hline & Índice \\
\hline Media & 0,47 \\
\hline DE & 0,34 \\
\hline
\end{tabular}


Como resumen de los resultados de la variable "Conocimientos culturales», es destacable que los resultados obtenidos en los índices son similares (medias: 0,50; 0,$55 ; 0,45$ y 0,47 ) Sin embargo, la variabilidad es generalmente alta en los indicadores que están relacionados directamente con el tema del texto.

\subsection{Variable «Conocimientos de traducción»}

En la Tabla 8 presentamos la definición, los indicadores medidos y los instrumentos utilizados en la variable «Conocimientos de traducción».

TABLA 8

Definición, indicadores e instrumentos de la variable "Conocimientos de traducción»

\begin{tabular}{|l|l|}
\hline \multicolumn{2}{|c|}{ CONOCIMIENTOS DE TRADUCCIÓN (PACTE 2014) } \\
\hline definición & $\begin{array}{l}\text { Los conocimientos implícitos del sujeto sobre los principios de la traducción y } \\
\text { sobre aspectos de la profesión. }\end{array}$ \\
\hline indicadores & $\begin{array}{l}\text { - Índice de dinamismo de los conocimientos de traducción: los conocimientos } \\
\text { implícitos de los sujetos sobre los principios de la traducción; } \\
\text { - Coeficiente de coherencia de los conocimientos de traducción: la coherencia de } \\
\text { los sujetos de la visión que tienen sobre los distintos aspectos de la traducción. } \\
\text { - Aceptabilidad. }\end{array}$ \\
\hline instrumentos & Cuestionario de conocimientos de traducción \\
\hline
\end{tabular}

\subsubsection{Indice de dinamismo de los conocimientos de traducción}

El rango de este índice va del -1 (concepción estática) al 1 (concepción dinámica). El índice se calcula con los datos recogidos del cuestionario de conocimientos de traducción. Para el cálculo, del cuestionario se seleccionaron cinco pares de ítems opuestos, relacionados con un aspecto concreto de la traducción como el encargo, el lector del texto de llegada o los métodos de traducción. A estos cinco pares se les asignó un valor numérico según la concepción del sujeto, marcada en las escalas de Likert (-1 a concepciones estáticas y 1 a concepciones dinámicas). En primer lugar, el índice se calcula para cada par de ítems y, en segundo lugar, para los cinco pares. Para más información acerca del método de cálculo de este indicador, v. PACTE (2014).

Las medias del índice de dinamismo de los conocimientos de traducción (Tabla 9) va desde el 0,25 hasta el 0,75, y la variabilidad es moderada en los cinco pares de ítems (desde 0.25 hasta 0.28 ). Los sujetos conciben el encargo de traducción y el lector del texto de llegada de manera dinámica y los métodos de traducción de manera más estática. El índice del grupo experimental es de 0,45, el mismo valor obtenido en el grupo de cuarto curso que participó en el experimento de ACT de PACTE (PACTE 2014: 104).

TABLA 9

Índice de dinamismo de los conocimientos de traducción

\begin{tabular}{|l|c|c|}
\hline & Media & DE \\
\hline Par 1 (encargo y lector del texto de llegada) & 0,75 & 0,28 \\
\hline Par 2 (métodos) & 0,37 & 0,25 \\
\hline Par 3 (métodos) & 0,62 & 0,25 \\
\hline Par 4 (métodos) & 0,25 & 0,28 \\
\hline Par 5 (métodos) & 0,25 & 0,28 \\
\hline Índice & 0,45 & 0,10 \\
\hline
\end{tabular}




\subsubsection{Coeficiente de coherencia de los conocimientos de traducción}

El rango de este coeficiente va del 0 (coherencia baja) al 1 (coherencia alta). En base a las respuestas a los cinco pares de ítems opuestos del cuestionario de conocimientos de traducción, se establecieron tres categorías de coherencia: 1 (totalmente coherente: la concepción del sujeto es totalmente estática o totalmente dinámica); 0,5 (parcialmente coherente: la concepción del sujeto es parcialmente estática o parcialmente dinámica), y 0 (totalmente incoherente). Para más información acerca del método de cálculo de este indicador, v. PACTE (2014).

El coeficiente de coherencia del grupo experimental (Tabla 10) es de 0,75, un valor mucho más alto que el obtenido por los estudiantes de cuarto curso que participaron en el experimento de ACT de PACTE: 0,39 (2014: 104). A pesar de que el coeficiente es más alto en la prueba piloto, la variabilidad $(0,29)$ es también muy alta.

TABLA 10

Coeficiente de coherencia de los conocimientos de traducción

\begin{tabular}{|l|c|}
\hline & Coeficiente \\
\hline Media & 0,75 \\
\hline DE & 0,29 \\
\hline
\end{tabular}

En los indicadores de esta variable se observa que tanto el grupo experimental de la prueba piloto como el grupo de cuarto curso del experimento de ACT obtienen resultados similares. A pesar de que los del grupo de la prueba piloto son más altos que los de los sujetos de cuarto de ACT, las desviaciones estándar indican que ambos son similares.

\subsection{Variable «Proyecto traductor»}

En la Tabla 11 presentamos la definición, los indicadores medidos y los instrumentos utilizados en la variable «Proyecto traductor».

TABLA 11

Definición, indicadores e instrumentos de la variable «Proyecto traductor»

\begin{tabular}{|l|l|}
\hline \multicolumn{2}{|c|}{ PROYECTO TRADUCTOR (PACTE 2014) } \\
\hline definición & $\begin{array}{l}\text { La representación mental y las expectativas del traductor de cómo debería ser la } \\
\text { traducción de un texto. }\end{array}$ \\
\hline indicadores & $\begin{array}{l}\text { - Índice de dinamismo del proyecto traductor global: el enfoque de los sujetos } \\
\text { para la traducción de un texto concreto; } \\
\text { - Índice de dinamismo del proyecto traductor de los problemas de traducción: el } \\
\text { enfoque de los sujetos en la traducción de las unidades de un texto. } \\
\text { - Índice de dinamismo de la traducción: la consistencia del concepto de la } \\
\text { traducción de los sujetos y su enfoque en la traducción de los problemas de } \\
\text { traducción específicos. } \\
\text { - Aceptabilidad. }\end{array}$ \\
\hline instrumentos & \begin{tabular}{l} 
Cuestionario de problemas de traducción \\
\hline
\end{tabular} \\
\hline
\end{tabular}




\subsubsection{Indice de dinamismo del proyecto traductor global}

El rango de este índice va desde el -1 (proyecto estático) hasta el 1 (proyecto dinámico). El índice se calcula a partir de las respuestas de los sujetos a la pregunta «¿Qué has considerado prioritario a la hora de traducir el texto?», del cuestionario de problemas de traducción. Las respuestas se dividen en categorías dinámicas y estáticas y se les asigna un 1 a las dinámicas y un -1 a las estáticas. Con estos valores se calcula la media para el grupo experimental. Para más información acerca del método de cálculo de este indicador, v. PACTE (2011a).

El índice de dinamismo del proyecto traductor global (Tabla 12) es totalmente dinámico (1,00). El grupo experimental es homogéneo en cuanto a la manera de enfrentarse al texto. El índice es incluso más alto que en el cuarto curso del experimento de ACT: 0,85 (PACTE 2015). Ambos grupos tienen un proyecto traductor similar.

TABLA 12

Índice de dinamismo del proyecto traductor global

\begin{tabular}{|l|c|}
\hline & Índice \\
\hline Media & 1,00 \\
\hline $\mathrm{DE}$ & 0,00 \\
\hline
\end{tabular}

4.4.2. Indice de dinamismo del proyecto traductor de los problemas de traducción

El rango de este índice va del -1 (concepción estática) hasta el 1 (concepción dinámica). El índice se calcula a partir de las respuestas de los sujetos a la pregunta «¿Qué has considerado prioritario a la hora de traducir el segmento?», del cuestionario de problemas de traducción. Las respuestas se dividen en categorías dinámicas y estáticas y se les asigna un 1 a las dinámicas y un -1 a las estáticas. Para más información acerca del método de cálculo de este indicador, v. PACTE (2011a).

La homogeneidad que se observa en el índice de dinamismo del proyecto traductor global no coincide con los resultados obtenidos en el índice de dinamismo del proyecto traductor de los problemas de traducción (Tabla 13), ya que la variabilidad es extremadamente alta (DE: 0,90$)$ y el índice tiende hacia una concepción estática a la hora de traducir los puntos ricos $(0,40)$.

Queremos destacar algunos aspectos a partir de los resultados. En primer lugar, Ostalgie se ha traducido desde una concepción muy dinámica, lo que podemos considerar una decisión lógica puesto que este punto rico se sitúa en el título del artículo y la propuesta de un diccionario bilingüe puede ser insuficiente para traducirlo de manera aceptable. En segundo lugar, algunos puntos ricos (Spreemetropole, Arbeiterund Bauernstaat y el punto especialmente rico) se han traducido desde una concepción dinámica, pero con una alta variabilidad (DE: 0,90). Finalmente, Grenzeruniformen se ha traducido desde una concepción estática $(0,20)$ y, comparando este resultado con la aceptabilidad de este punto rico (0,00; Tabla 2), podría interpretarse que es una concepción que no funciona para llegar a soluciones aceptables en este punto rico en concreto. 
TABLA 13

Índice de dinamismo del proyecto traductor de los problemas de traducción

\begin{tabular}{|l|c|c|}
\hline & Media & DE \\
\hline Ostalgie (cultura lingüística) & 1,00 & 0,00 \\
\hline Spreemetropole (medio natural) & 0,60 & 0,90 \\
\hline Grenzeruniformen (patrimonio cultural) & 0,20 & 1,10 \\
\hline Arbeiter- und Bauernstaat (cultura social) & 0,60 & 0,90 \\
\hline Punto especialmente rico & 0,60 & 0,90 \\
\hline Grupo experimental & 0,40 & 0,90 \\
\hline
\end{tabular}

\subsubsection{Indice de dinamismo de la traducción}

Este índice va desde el 0 (concepción estática) hasta el 1 (concepción dinámica). Para este indicador se calcula la media entre el índice de dinamismo de los conocimientos de traducción, el índice de dinamismo del proyecto traductor global y el índice de dinamismo del proyecto traductor de los problemas de traducción.

Ya hemos mencionado la diferencia en la homogeneidad entre el índice de dinamismo del proyecto traductor global y el índice de dinamismo del proyecto traductor de los problemas de traducción y se confirma con los resultados del índice de dinamismo de la traducción (Tabla 14).

TABLA 14

Índice de dinamismo de la traducción

\begin{tabular}{|l|c|}
\hline & Índice \\
\hline Media & 0,55 \\
\hline DE & 0,26 \\
\hline
\end{tabular}

En esta variable se observa que el grupo experimental de la prueba piloto tiene una concepción dinámica de la traducción, pero cuando se trata de traducir los puntos ricos el grupo tiende hacia una concepción más estática, incluso en aquellos puntos ricos en los cuales no lleva a una solución aceptable.

\subsection{Variable «Identificación y resolución de problemas de traducción»}

En la Tabla 15 presentamos la definición, los indicadores medidos y los instrumentos utilizados en la variable «Identificación y resolución de problemas de traducción». 
TABLA 15

Definición, indicadores e instrumentos de la variable «Identificación y resolución de problemas de traducción»

\begin{tabular}{|l|l|}
\hline \multicolumn{2}{|c|}{ IDENTIFICACIÓN Y RESOLUCIÓN DE LOS PROBLEMAS DE TRADUCCIÓN } \\
DE ÍNDOLE CULTURAL
\end{tabular}

\subsubsection{Indice de identificación de los problemas de traducción de índole cultural}

Este índice va desde el 0 (ningún problema identificado) hasta el 100\% (todos los problemas identificados). Los datos para este indicador se obtienen de la pregunta para cada punto rico "¿Te ha planteado dificultades el elemento subrayado?», del cuestionario de problemas de traducción. Este indicador se calcula para cada punto rico y para el grupo experimental en general. Para más información acerca del método de cálculo de este indicador, v. PACTE (2011b).

La identificación de los puntos ricos como problemas de traducción (Tabla 16) va desde el $80 \%$ hasta el $100 \%$. La identificación en el caso del grupo experimental asciende al $88 \%$.

TABLA 16

Identificación de los puntos ricos como problemas de traducción

\begin{tabular}{|l|c|}
\hline Punto rico & Porcentaje \\
\hline Ostalgie (cultura lingüística) & 80,00 \\
\hline Spreemetropole (medio natural) & 80,00 \\
\hline Grenzeruniformen (patrimonio cultural) & 100,00 \\
\hline Arbeiter- und Bauernstaat (cultura social) & 100,00 \\
\hline Punto especialmente rico & 80,00 \\
\hline Grupo experimental & 88,00 \\
\hline
\end{tabular}

\subsubsection{Caracterización de problemas de traducción de índole cultural}

Los datos para este indicador se obtienen de la pregunta «¿Qué dificultades generales tiene la traducción de este texto?», del cuestionario de problemas de traducción. Los sujetos debían seleccionar hasta tres categorías y ordenarlas de mayor a menor dificultad. Para este indicador se calcula el porcentaje válido para cada categoría.

De acuerdo con la categorización de los sujetos (Figura 2), el primer problema de traducción es de índole cultural (80\%), el segundo lo causan las dificultades léxicas y morfosintácticas de reexpresión (60\%) y el tercero las dificultades a la hora de producir un texto coherente y estilísticamente adecuado (80\%). La variabilidad en los resultados es baja puesto que los sujetos caracterizan los problemas en un orden similar. 
FIGURA 2

Caracterización de problemas de traducción de índole cultural

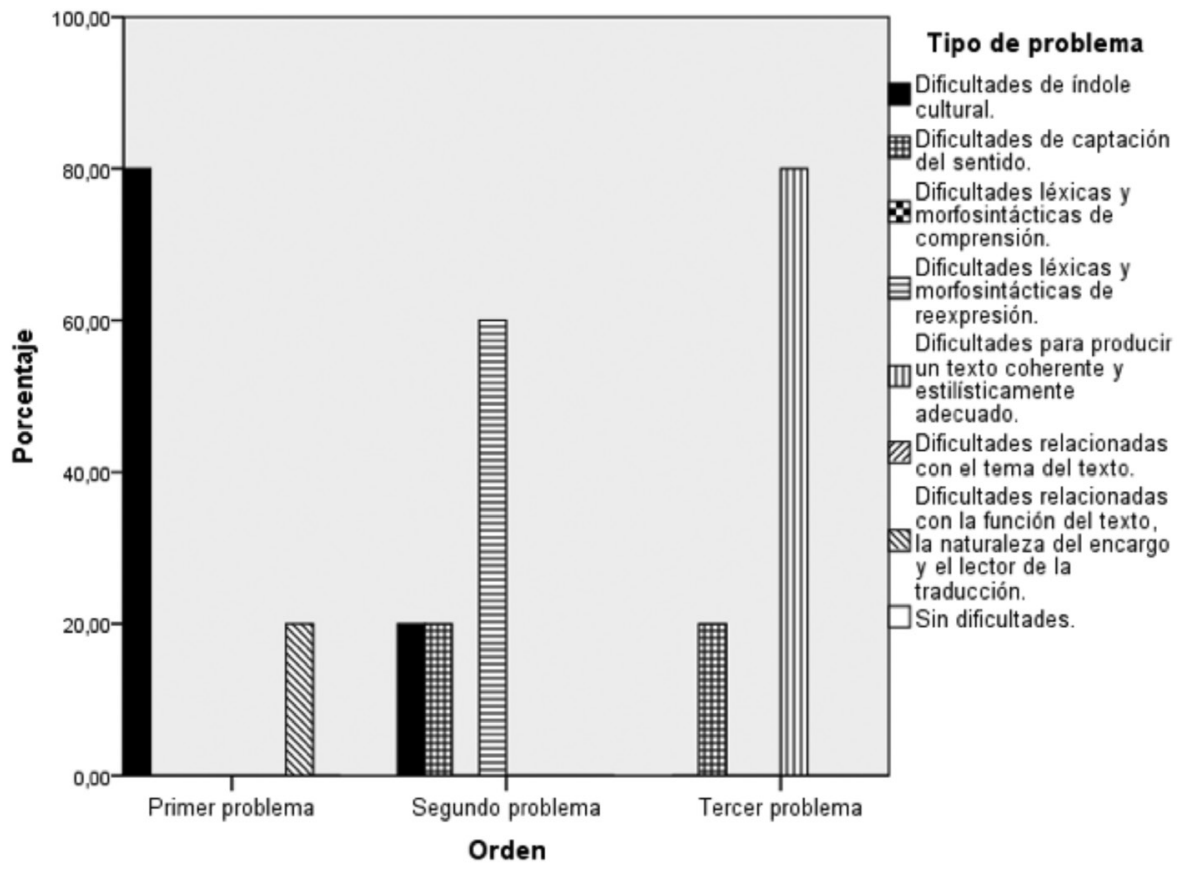

\subsubsection{Caracterización de las dificultades de los puntos ricos}

Los datos para este indicador se obtienen de la pregunta para cada punto rico «¿Qué tipo de dificultades de traducción plantea el elemento subrayado?», del cuestionario de problemas de traducción. Para este indicador se calcula el porcentaje válido para cada categoría.

Los resultados (Tabla 17) muestran que Ostalgie y el punto especialmente rico causan dificultades de reexpresión (80\%). Grenzeruniformen y Arbeiter- und Bauernstaat causan dificultades tanto de comprensión como de reexpresión (40\% y $60 \%$ respectivamente en Grenzeruniformen y $60 \%$ y $40 \%$ respectivamente en Arbeiter-und Bauernstaat). Spreemetropole causa dificultades de comprensión (40\%).

TABLA 17

Caracterización de las dificultades de los puntos ricos

\begin{tabular}{|l|c|c|c|c|c|}
\hline & Ostalgie & Spreemetropole & Grenzeruniformen & $\begin{array}{c}\text { Arbeiter-und } \\
\text { Bauernstaat }\end{array}$ & $\begin{array}{c}\text { Punto especial- } \\
\text { mente rico }\end{array}$ \\
\hline $\begin{array}{l}\text { Dificultades de } \\
\text { comprensión }\end{array}$ & 20,00 & 40,00 & 40,00 & 60,00 & 20,00 \\
\hline $\begin{array}{l}\text { Dificultades de } \\
\text { reexpresión }\end{array}$ & 80,00 & 20,00 & 60,00 & 40,00 & 80,00 \\
\hline $\begin{array}{l}\text { Otras } \\
\text { dificultades }\end{array}$ & 0,00 & 40,00 & 0,00 & 0,00 & 0,00 \\
\hline
\end{tabular}




\subsubsection{Coeficiente de satisfacción con la traducción}

El rango de este coeficiente va desde el 0 (satisfacción baja) hasta el 1 (satisfacción alta). Los datos para este indicador se obtienen de la pregunta para cada punto rico «¿Estás satisfecho/a con tu solución?», del cuestionario de problemas de traducción. Para el cálculo, se asigna un valor numérico a las respuestas de los sujetos (sí = 1 ; no $=0$, y parcialmente $=0,5)$. Este indicador se calcula para cada punto rico y para el grupo experimental. Para más información acerca del método de cálculo de este indicador, v. PACTE (2011b).

En los puntos ricos, el coeficiente de satisfacción (Tabla 18) va desde el 0,20 hasta el 1,00. El valor más alto se da en Spreemetropole (1,00), que también es el punto rico con mayor aceptabilidad junto con Ostalgie $(0,60)$. La satisfacción más baja se da en Grenzeruniformen $(0,20)$. Este punto rico obtuvo la aceptabilidad más baja $(0,00)$. En cuanto a la variabilidad, la desviación es más bien alta (desde 0,45 hasta 0,55 sin incluir Spreemetropole).

El coeficiente de satisfacción global es de 0,48 y la variabilidad es alta $(0,30)$. La satisfacción global es más alta que la aceptabilidad (0,37, Tabla 2).

TABLA 18

Coeficiente de satisfacción

\begin{tabular}{|l|c|c|}
\hline Punto rico & Índice & $\mathrm{DE}$ \\
\hline Ostalgie (cultura lingüística) & 0,40 & 0,55 \\
\hline Spreemetropole (medio natural) & 1,00 & 0,00 \\
\hline Grenzeruniformen (patrimonio cultural) & 0,20 & 0,45 \\
\hline Arbeiter- und Bauernstaat (cultura social) & 0,40 & 0,55 \\
\hline Punto especialmente rico & 0,40 & 0,55 \\
\hline Grupo experimental & 0,48 & 0,30 \\
\hline
\end{tabular}

\subsubsection{Indice de percepción de la dificultad de la traducción}

Este índice va desde el 0 (dificultad baja) hasta el 1 (dificultad alta). Los datos para este indicador se obtienen de la pregunta «¿Qué grado de dificultad tiene la traducción de este texto?», del cuestionario de problemas de traducción. Los sujetos marcaron la dificultad del texto en una escala del 1 al 10. Los valores se recalcularon en una escala del 0 al 1. Este indicador se calcula para el grupo experimental.

La dificultad de la traducción (Tabla 19) se considera media $(0,64)$, aunque la variabilidad es alta $(0,89)$.

TABLA 19

Índice de percepción de la dificultad de la traducción

\begin{tabular}{|l|c|}
\hline & Índice \\
\hline Media & 0,64 \\
\hline DE & 0,89 \\
\hline
\end{tabular}

En esta variable se observa que los puntos ricos se identifican como problemas de traducción de índole cultural y el texto causa dificultades relacionadas con aspectos culturales. En general, el texto se percibe como moderadamente difícil. Los 
puntos ricos causan problemas tanto de comprensión como de reexpresión. La satisfacción con las soluciones propuestas es más alta que la aceptabilidad que obtienen. Algunos índices presentan una variabilidad alta. Esto indica la necesidad de correlacionar los resultados de varios indicadores para intentar explicar este hecho.

\subsection{Variable «Toma de decisiones»}

En la Tabla 20 presentamos la definición, los indicadores medidos y los instrumentos utilizados en la variable «Toma de decisiones».

TABLA 20

Definición, indicadores e instrumentos de la variable «Toma de decisiones»

\begin{tabular}{|l|l|}
\hline \multicolumn{2}{|c|}{ TOMA DE DECISIONES (PACTE 2014) } \\
\hline definición & $\begin{array}{l}\text { Las decisiones tomadas durante el proceso traductor que afectan al uso de } \\
\text { recursos cognitivos automatizados y no automatizados (apoyo interno) y el uso de } \\
\text { recursos de documentación (apoyo externo) (Alves 1995; 1997). }\end{array}$ \\
\hline indicadores & $\begin{array}{l}\text { - Secuencias de acciones: el tipo de apoyo utilizado por los sujetos para la } \\
\text { traducción de los puntos ricos; } \\
\text { - Aceptabilidad. }\end{array}$ \\
\hline instrumentos & Traducciones y grabaciones de pantalla \\
\hline
\end{tabular}

\subsubsection{Secuencias de acciones}

Se trata de un indicador categórico cuyos resultados se ofrecen en porcentajes. Las siguientes categorías se emplean para describir las secuencias de acciones usadas para solucionar los problemas de traducción:

- apoyo interno (AI). La solución definitiva se basa exclusivamente en apoyo interno; no hay consultas antes de la solución definitiva. Puede ser automatizado (se llega a la solución automáticamente, casi sin pensar) o no automatizado (se llega a la solución pensando);

- predominio de apoyo interno (PAI). La solución definitiva se basa en apoyo interno, así como en cualquier consulta que incluya recursos bilingües cuya solución no se adopte en la traducción;

- predominio de apoyo externo (PAE). La solución definitiva se basa en apoyo externo, así como en cualquier consulta que incluya recursos bilingües cuya solución se adopte en la traducción;

- apoyo externo (AE). La solución definitiva se basa exclusivamente en consultas de recursos bilingües cuya solución se adopta en la traducción.

Los datos se obtienen a partir de las grabaciones de Camtasia. Para más información acerca del método de cálculo de este indicador, (PACTE 2009).

Los tipos de secuencias utilizados (Tabla 21) muestran que se llegó a las soluciones utilizando principalmente predominio de apoyo interno y predominio de apoyo externo (39,10\% en ambas secuencias). En un 17,40\% de las soluciones se utilizó apoyo externo. Solamente se observó una solución $(4,30 \%)$ con apoyo interno (automatizado). 
TABLA 21

Tipos de secuencias

\begin{tabular}{|l|c|}
\hline Tipo de secuencia & Porcentaje válido \\
\hline Apoyo interno (automatizado) & 4,30 \\
\hline Predominio de apoyo interno & 39,10 \\
\hline Predominio de apoyo externo & 39,10 \\
\hline Apoyo externo & 17,40 \\
\hline
\end{tabular}

Si se observan los tipos de secuencias para cada punto rico (Tabla 22), destaca que Ostalgie se soluciona con predominio de apoyo externo (60\%) o apoyo externo (40\%). En Grenzeruniformen y en el punto especialmente rico, se usan tanto predominio de apoyo interno como externo. Spreemetropole se resuelve usando solamente secuencias de acciones relacionadas con el apoyo interno (20\% en apoyo interno y $80 \%$ en predominio de apoyo interno). En Arbeiter- und Bauernstaat se usa apoyo externo $(66,70 \%)$ y predominio de apoyo interno $(33,30 \%)$.

TABLA 22

Tipos de secuencias para cada punto rico

\begin{tabular}{|l|c|c|}
\hline Punto rico & Secuencia & Porcentaje válido \\
\hline \multirow{2}{*}{ Ostalgie (cultura lingüística) } & AE & 40,00 \\
\cline { 2 - 3 } & PAE & 60,00 \\
\hline \multirow{2}{*}{ Spreemetropole (medio natural) } & AI & 20,00 \\
\cline { 2 - 3 } & PAI & 80,00 \\
\hline \multirow{2}{*}{ Grenzeruniformen (patrimonio cultural) } & PAE & 80,00 \\
\cline { 2 - 3 } & PAI & 20,00 \\
\hline \multirow{2}{*}{ Arbeiter- und Bauernstaat (cultura social) } & AE & 66,70 \\
\hline \multirow{2}{*}{ Punto especialmente rico } & PAI & 33,30 \\
\cline { 2 - 3 } & PAE & 40,00 \\
\cline { 2 - 3 } & PAI & 60,00 \\
\hline
\end{tabular}

En esta variable se observa que utilizar exclusivamente los conocimientos previos sobre los puntos ricos o los recursos de documentación no es suficiente para solucionar los puntos ricos de manera adecuada, puesto que las secuencias más frecuentes son la mezcla de ambos: predominio de apoyo interno y predominio de apoyo externo. En el grupo experimental, cada punto rico se ha resuelto utilizando como mínimo dos tipos de secuencias distintas. Esto demuestra que los puntos ricos se solucionan de maneras distintas según las necesidades del sujeto.

\section{Conclusiones}

En esta sección se presentan las conclusiones más relevantes ordenadas según los aspectos metodológicos evaluados.

\subsection{Criterios de selección de la muestra}

Los criterios de selección de la muestra han funcionado porque se ha extraído una muestra con características similares a las del universo de estudio: en los indicadores 
índice de dinamismo de los conocimientos de traducción, coeficiente de coherencia de los conocimientos de traducción e índice de dinamismo del proyecto traductor global, la muestra de la prueba piloto ha obtenido resultados similares al grupo de cuarto curso del experimento de ACT. Ambas tienen unos conocimientos implícitos sobre la traducción similares y parten de una concepción dinámica a la hora de traducir.

Sin embargo, la variabilidad es alta en muchos indicadores, sobre todo en aquellos que están relacionados con el proceso traductor. En el experimento, estos indicadores deberán cruzarse con otros con el fin de esclarecer qué causa esta variabilidad.

\subsection{Funcionamiento de los instrumentos de recogida de datos}

Los instrumentos han funcionado adecuadamente, aunque el cuestionario de conocimientos culturales necesita validarse con más procedimientos (Olalla-Soler y Neunzig, en proceso). El texto se percibe como moderadamente difícil y, en general, los sujetos tienen conocimientos medios acerca del tema del texto. El cuestionario de conocimientos de traducción, ya validado por PACTE (2014), también ha funcionado correctamente. El cuestionario de problemas de traducción, adaptado de PACTE (2011b), ha recopilado información pertinente para el análisis. El documento para la identificación y caracterización de los culturemas ha servido para recopilar datos relevantes para el análisis de la identificación y caracterización de los culturemas.

\subsection{Adecuación de los puntos ricos}

Los puntos ricos seleccionados son adecuados para el análisis de la competencia cultural, puesto que en la variable «Toma de decisiones» los puntos ricos se resuelven combinando apoyo interno y externo y, además, los sujetos utilizan secuencias de acciones distintas para solucionarlos dependiendo de sus necesidades.

Los puntos ricos se identifican como problemas de traducción y se categorizan como problemas de índole cultural. Por ello, la naturaleza cultural de los puntos ricos queda asegurada. En general, los puntos ricos no son solucionables de manera automática. Esto es importante para recopilar datos en nuestro estudio, ya que suele obligar a los sujetos a vincular la competencia cultural con las subcompetencias de la competencia traductora para llegar a soluciones aceptables. Puesto que los puntos ricos causan dificultades tanto de comprensión como de reexpresión, ambos aspectos quedan cubiertos en el experimento.

El texto causa dificultades de carácter cultural, se percibe como moderadamente difícil y la satisfacción con las soluciones es más bien baja.

\subsection{Tareas experimentales}

Para algunos indicadores relacionados con la traducción, no fue posible recopilar datos de los dos últimos puntos ricos (Arbeiter- und Bauernstaat y el punto especialmente rico). Al estar ubicados hacia el final del texto, algunos sujetos no tuvieron tiempo para finalizar la traducción y, por ello, se ha revisado el tiempo asignado a cada tarea y se ha ampliado el tiempo destinado a la traducción. 


\subsection{Variables}

Las variables seleccionadas para la prueba piloto han aportado resultados pertinentes para nuestro estudio. También se ha identificado la necesidad de realizar cruzamientos entre indicadores para explicar la variabilidad de los datos y para establecer relaciones entre variables.

Después de revisar los resultados y de relacionarlos con el modelo de competencia cultural propuesto en la sección 2.3, hemos observado que no se han recogido datos acerca de los componentes actitudinales a pesar de estar incluidos en el modelo. Por ello, se ha creado una nueva variable: «Componentes actitudinales de la competencia cultural del traductor».

Puesto que en nuestro modelo estas actitudes se basan en constructos validados empíricamente como el de inteligencia cultural y el de eficacia multicultural, se decide utilizar como instrumentos de recogida de datos para esta variable los test Cultural Intelligence Scale (Ang, van Dyne et al 2007) y Multicultural Personality Questionnaire (van der Zee y van Oudenhoven 2000). Estos cuestionarios aportan información pertinente acerca de las actitudes que favorecen el relativismo cultural y la sensibilización cultural y además han sido probados y validados por distintos autores (Rushton y Irwing 2009; Imai y Gelfand 2010; Matsumoto y Hwang 2013).

\subsection{Comentarios finales}

Aunque las hipótesis relacionadas con la evolución de la adquisición de la competencia cultural no pueden verificarse con los datos de un solo curso, de los resultados comentados se desprende que:

- los conocimientos culturales de la muestra son relativamente bajos para tratarse de cuarto curso. Dado que la muestra de la prueba piloto proviene de estudiantes de alemán como segunda lengua extranjera, esta ha recibido una enseñanza orientada hacia la adquisición de la lengua y no tanto hacia la adquisición de aspectos culturales. Si bien es cierto que las asignaturas de alemán como segunda lengua extranjera no pueden abarcar todos estos contenidos, consideramos que deberían desarrollarse las habilidades de adquisición de conocimientos culturales y las contrastivas en las asignaturas de traducción de alemán como segunda lengua extranjera con el fin de minimizar las dificultades culturales a las cuales los estudiantes deben hacer frente al no poseer conocimientos culturales suficientes.

- En el caso de las habilidades de adquisición de conocimientos culturales, nuestra muestra de cuarto curso presenta una característica destacable: al usarse mayormente secuencias de acciones para la documentación que incluyen recursos que no se reducen exclusivamente a los diccionarios bilingües, se desprende que los estudiantes son conscientes de que el uso exclusivo de los diccionarios bilingües no es necesariamente la mejor opción para la traducción de referencias culturales. A pesar de que la eficacia de uso de los recursos documentales no se puede medir con los indicadores que hemos usado en la prueba piloto, consideramos que es importante desarrollar estrategias de uso de recursos para mejorar la eficacia, como identificar necesidades documentales, planificar búsquedas, utilizar procedimientos para obtener resultados más pertinentes en las búsquedas, evaluar fuentes de documentación, etc.

- Consideramos que las habilidades contrastivas deberían trabajarse con más detenimiento dados los resultados obtenidos en nuestra muestra de cuarto curso. En pri- 
mer lugar, observamos que las habilidades contrastivas para la resolución de problemas de traducción de índole cultural no están especialmente desarrolladas, puesto que la aceptabilidad del grupo experimental es de 0,37 sobre 1 . En segundo lugar, solamente la mitad de puntos ricos se han identificado como culturemas. Esto significa que los estudiantes de la muestra no son capaces de identificar la mayoría de referencias culturales de un texto, siendo la identificación un paso esencial para la adecuada resolución de los problemas de traducción de índole cultural. Creemos que los estudiantes deben desarrollar habilidades y estrategias para identificar culturemas, para solucionar problemas de traducción de índole cultural y, sobre todo, para evaluar sus propias soluciones.

Si bien es cierto que en este estudio experimental se manipula una gran cantidad de variables y se mide una amplia variedad de indicadores, el diseño presenta ciertas limitaciones relacionadas principalmente con los elementos estudiados como puntos ricos: los culturemas. A pesar de seleccionar culturemas de distinta naturaleza para así cubrir todos los ámbitos culturales de Molina (2001), estos representan uno de los posibles niveles culturales que se dan en una cultura. Katan (2009), basándose en Hall (1959), clasifica jerárquicamente estos niveles según el grado de visibilidad de los aspectos que contienen. Los culturemas se sitúan en el nivel de «technical culture», que corresponde al más visible.

En nuestro estudio, pretendemos contrarrestar la limitación que apuntamos mediante la inclusión de los test psicométricos Cultural Intelligence Scale y Multicultural Personality Questionnaire con el objetivo de obtener datos de niveles menos visibles.

Mediante los datos recogidos en el estudio experimental revisado, observaremos con mayor detalle la adquisición de estos componentes de la competencia cultural del traductor y formularemos propuestas didácticas para ayudar a mejorar su adquisición.

Entre las propuestas que pretendemos desarrollar, destacamos una batería de tareas para desarrollar cada uno de los componentes de la competencia cultural. El objetivo de esta propuesta didáctica es el de ofrecer tareas que respondan a las necesidades formativas específicas de la competencia cultural en cada curso. Para ello, será necesario observar las carencias de los grupos de muestra del experimento. En relación con esta propuesta didáctica, pretendemos también elaborar un primer esbozo de nivelación de la adquisición de la competencia cultural basándonos en los resultados del experimento, en la propuesta de Katan (2008) y en la de PICT (2012). Ya fuera del marco de este estudio experimental, se revisará este esbozo con documentación de otras universidades y, de esta manera, se propondrá una nivelación basada en datos empíricos del experimento.

Una propuesta que ya hemos desarrollado es un compendio de instrumentos de evaluación de la adquisición de la competencia cultural (Hurtado Albir y Olalla-Soler, 2016). En él se incluyen nueve instrumentos con las tareas evaluadoras y los aspectos evaluables relacionados.

\section{AGRADECIMIENTOS}

Deseo agradecer a Micah Reverdy, traductor y corrector profesional, su labor de revisión del resumen en francés. 


\section{NOTAS}

1. En nuestro estudio partimos de la definición de PACTE para el concepto de aceptabilidad. Sin embargo, el primero en definir el concepto de aceptabilidad es Toury (1980), aunque con una concepción distinta a la de PACTE. Según Toury, la aceptabilidad es un método de traducción en el cual priman las normas de la cultura receptora frente a las de la cultura de partida.

\section{REFERENCIAS BIBLIOGRÁFICAS}

Alves, Fabio. (1995): Zwischen Schweigen und Sprechen: Wie bildet sich eine transkulturelle Brücke? Eine Analyse von Übersetzungsvorgängen zwischen portugiesischen und brasilianischen Übersetzern. Hamburg: Dr. Kovac.

Alves, Fabio. (1997): A formação de tradutores a partir de uma abordagem cognitiva: reflexões de um projeto de ensino. TradTerm. Revista do Centro Interdepartamental de Tradução e Terminologia. 4(2):19-40.

ANG, Soon, van Dyne, Linn, Кон, Christine et al. (2007): Cultural intelligence: Its measurement and effects on cultural judgment and decision making. Cultural adaptation and task performance. Management and Organization Review. 3(3):335-371.

Arasaratnam, Lily A. (2007): Research in intercultural communication competence. Journal of International Communication. 13(2):66-73.

Byram, Michael (1997): Teaching and Assessing Intercultural Communicative Competence. Assessment. United Kingdom: Multilingual Matters.

DeARDorfF, Darla K. (2006): Identification and assessment of intercultural competence as a student outcome of internationalization. Journal of Studies in International Education. 10(3):241-266.

Fantini, Alvino E. (1995): Language, culture, and world view: Exploring the nexus. International Journal of Intercultural Relations. 19(2):143-153.

Hall, Edward T. (1959): The Silent Language. New York: Doubleday.

HARris, Marvin (2011): Antropología Cultural. Madrid: Alianza Editorial.

Hurtado Albir, Amparo (2017): Researching Translation Competence by PACTE Group. Amsterdam/Philadelphia: John Benjamins.

Hurtado Albir, Amparo y Olalla-Soler, Christian (2016): Procedures for assessing the acquisition of cultural competence in translator training. The Interpreter and Translator Trainer. 10(3):318-342.

ImaI, Lynn y Gelfand, Michele J. (2010): The culturally intelligent negotiator: The impact of cultural intelligence (CQ) on negotiation sequences and outcomes. Organizational Behavior and Human Decision Processes. 112(2):83-98.

Katan, David (2008): University training, competencies and the death of the translator. Problems in professionalizing translation and in the translation profession. In: Maria Teresa MusACChio y Genevieve Henrot, eds. Tradurre: Formazione e Professione. Padova: CLEUP, 113-140.

Katan, David (2009): Culture. In: Mona Baker y Gabriela Saldanha, eds. Routledge Encyclopedia of Translation Studies. $2^{\mathrm{a}}$ ed. London/New York: Routledge, 70-74.

King, Patricia M. y Baxter Magolda, Marcia B. (2005): A developmental model of intercultural Maturity. Journal of College Student Development. 46(6):571-592.

KUPKA, Bernd (2008): Creation of an instrument to assess intercultural communication competence for strategic international human resource management. Tesis de doctorado no publicada. Otago: University of Otago.

Matsumoto, David y Hwang, Hyisung C. (2013): Assessing cross-cultural competence: A review of available tests. Journal of Cross-Cultural Psychology. 44(6):849-873.

Molina, Lucía (2001): Análisis descriptivo de la traducción de los culturemas árabe-español. Tesis doctoral no publicada. Bellaterra: Universitat Autònoma de Barcelona.

NoRD, Christiane (1994): It's tea time in Wonderland. Culture-markers in fictional texts. In: Heiner PÜRsCHel et al, eds. Intercultural Communication. (17th International L.A.U.D. Symposium, Duisburg, 1992). Frankfurt etc.: Peter Lang, 523-538. 
Nord, Christiane (1997): Translating as a Purposeful Activity. Functionalist Approaches Explained. Machester: St Jerome.

OкsA AR, Els (1988): Kulturemtheorie. Ein Beitrag zur Sprachenverwendungsforschung. Gotinga: Vanderbhoeck \& Ruprecht.

Olalla-Soler, Christian (2015): An experimental study into the acquisition of the cultural competence in translator training. Research design and methodological issues. Translation \& Interpreting. 7(1):86-110. <http://www.trans-int.org/index.php/transint/article/view/ $338 / 186>$.

Olalla-Soler, Christian y Hurtado Albir, Amparo (2014): Estudio empírico de la traducción de los culturemas según el grado de adquisición de la competencia traductora. Un estudio exploratorio. Sendebar. 25:9-38.

Olalla-Soler, Christian y Neunzig, Wilhelm (en proceso). Measuring trainee translators' knowledge of German culture: Design and validation of a cultural declarative knowledge questionnaire.

PACTE (2003): Building a translation competence model. In: Fabio Alves, ed. Triangulating Translation: Perspectives in Process Oriented Research. Amsterdam: John Benjamins, 43-66.

PACTE (2009): Results of the validation of the PACTE translation competence model: Acceptability and decision making. Across Languages and Cultures. 10:207-230.

PACTE (2011a): Results of the validation of the PACTE translation competence model: Translation problems and translation competence. In: Cecilia Alvstad, Adelina Hild et al, eds. Methods and Strategies of Process Research: Integrative Approaches in Translation Studies. Amsterdam: John Benjamins, 317-343.

PACTE (2011b): Results of the validation of the PACTE translation competence model: Translation project and dynamic translation index. In: Sharon O'Brien, ed. Cognitive Explorations of Translation. London: Continuum Intl Pub Group, 30-56.

PACTE (2014): First results of PACTE group's experimental research on translation competence acquisition: The acquisition of declarative knowledge of translation. MonTI. Monografías de Traducción e Interpretación. Special issue 1:85-115.

PACTE (2015): Results of PACTE's experimental research on the acquisition of translation competence: The acquisition of declarative and procedural knowledge in translation. The dynamic translation index. Translation Spaces. (4)1:29-53.

PICT (actualizado en septiembre de 2012): Intercultural Competence Curriculum Framework. Consultado el 19 de abril de 2016, http://www.pictllp.eu/download/PICT_Curriculum_Framework.pdf.

Poyatos, Fernando (1976): Man beyond Words: Theory and Methodology of Non-verbal Communication. New York: New York State English Council.

Rathje, Stefanie (2007): Intercultural competence: The status and future of a controversial concept. Language and Intercultural Communication. 7(4):254-266.

Rushton, J. Philippe y Irwing, Paul (2009): A general factor of personality in the Comrey Personality Scales, the Minnesota Multiphasic Personality Inventory-2, and the Multicultural Personality Questionnaire. Personality and Individual Differences. 46(4):437-442.

SitzBerg, Brian H. y Changnon, Gabrielle (2009): Conceptualizing intercultural competence. In: Darla K. Deardorff, ed. The SAGE Handbook of Intercultural Competence. California: SAGE Publications, 2-52.

Toury, Gideon (1980): In Search of a Theory of Translation. Tel Aviv: The Porter Institute for Poetics and Semiotics.

VAN DeR Zee, Karen I. y van Oudenhoven, Jan P. (2000): The multicultural personality questionnaire: a multidimensional instrument of multicultural effectiveness. European Journal of Personality. 14(4):291-309.

Vermeer, Hans J. (1983): Aufsätze zur Translationstheorie. Heidelberg. 